\title{
Primary cutaneous histoplasmosis developed in the penis of an immunocompetent patient ${ }^{*}$
}

\author{
Mauricio Paixão \\ João Avancini ${ }^{3}$
}

\author{
Hélio Amante Miot ${ }^{2}$ \\ Walter Belda Júnior ${ }^{3}$
}

\begin{abstract}
A 70-year-old male presenting a 3-month history of genital painless erythematous nodules in the balanopreputial sulcus was referred to our service. Histopathological exam presented a chronic dermatitis with epithelioid granulomas and Grocott staining revealed numerous fungal structures with a suggestive morphology of Histoplasma sp. Cultures evidenced Histoplasma capsulatum var. capsulatum. Treatment with oral itraconazole led to complete remission of lesions.
\end{abstract}

Keywords: Bacterial infections and mycoses; Genital diseases, male; Genitalia, male; Histoplasmosis; Infection; Mycoses

\section{INTRODUCTION}

Histoplasma capsulatum is commonly found in soil contaminated by feces of birds and bats. Most human infections are subclinical. Symptomatic cases are usually manifested as self-limiting respiratory tract infections and disseminated infections are primarily associated with immunosuppression. ${ }^{1}$ Skin lesions occur in $4-11 \%$ of patients and result from secondary invasion of the skin in patients with disseminated infection. Primary cutaneous histoplasmosis is an extremely rare clinical entity and previously reported cases were mostly related to traumatic inoculation. ${ }^{2,3}$

\section{CASE REPORT}

We report the case of a 70-year-old male patient, white, from the urban area of Sao Paulo (Brazil), presenting a 3-month history of genital painless erythematous nodules in the balanopreputial sulcus, measuring $2.0 \mathrm{~cm}$ and $0.7 \mathrm{~cm}$ in diameter (Figures 1-3). Adenopathy and systemic symptom were absent. Type II Diabetes mellitus was diagnosed 15 years ago (using Metformin and Glimepiride), hypertension (using Captopril and Atenolol) and cigarette smoking for 50 years. He denied previous local trauma, but frequently traveled to a farm (countryside of the state of Sao Paulo), where he usually spent time cleaning one of the rooms where he often found bats. Patient also bred birds at home. Histopathological exam presented chronic dermatitis with epithelioid granulomas characterized by the presence of epithelioid or vacuolated histiocytes, some of which were grouped into giant cells, often phagocytizing the etiologic agent.

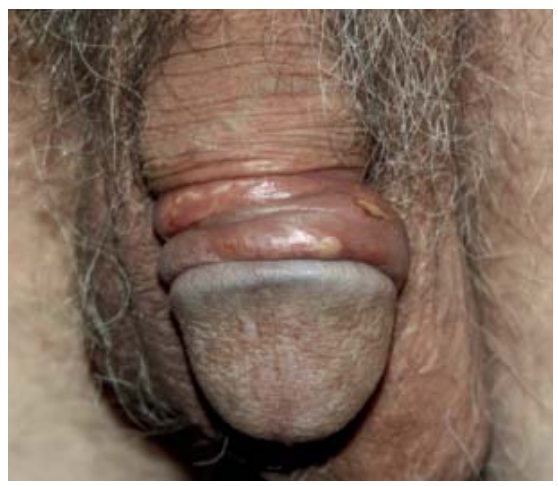

Figure 1:

Clinical

Picture

Received on 25.10.2013.

Approved by the Advisory Board and accepted for publication on 12.11.2013.

* Work performed at the Teaching Hospital (Hospital das Clínicas), Universidade de São Paulo (HC-FMUSP) - São Paulo (SP), Brazil.

Financial Support: none

Conflict of Interests: none 


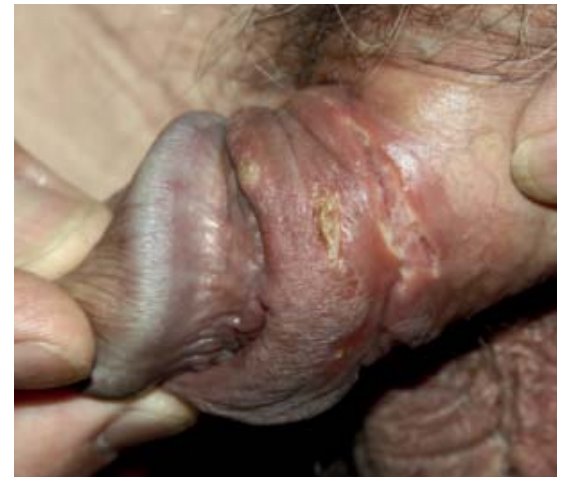

FIGURE 2:

Clin ic a 1

Picture

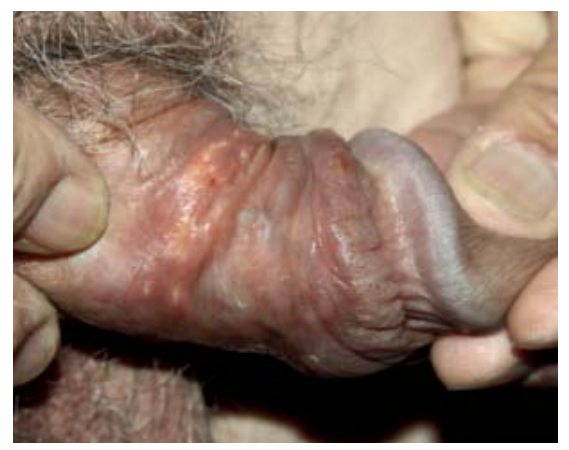

FIGURE 3

Clin i c a 1

Picture

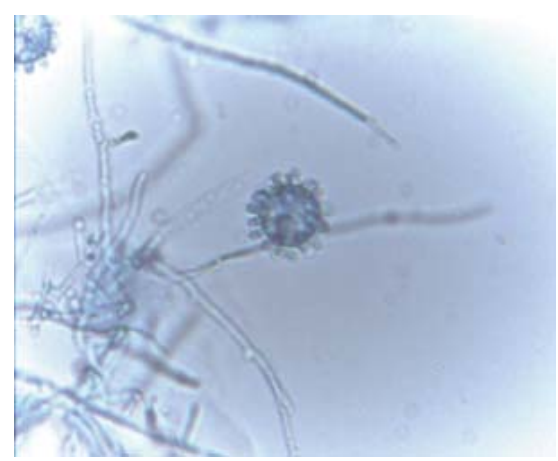

FiguRe 4:

Microculture presenting Histoplasma capsulatum

Grocott staining revealed numerous fungal structures with a suggestive morphology of Histoplasma sp. Sabouraud-agar with chloramphenicol and later Mycosel-agar cultures evidenced Histoplasma capsulatum var. capsulatum (Figure 4). Blood tests resulted negative for HIV 1/2, HCV, HBV, VDRL and ANA; C3, C4, ESR, total protein fractions, protein electrophoresis, electrolytes, fasting glucose, renal function test and $C B C$ were all within normal values. Serological tests (immunodiffusion and counterimmunoelectrophoresis) directed to Histoplasma capsulatum were negative. Chest radiography and computed tomography were performed and no changes were observed in the lung parenchyma. Treatment with Itraconazole $200 \mathrm{mg}$-day was prescribed and impor- tant regression of cutaneous lesions was noted after 1 month. A new biopsy with specimen culture was performed in the sixth month of treatment, presenting caseous necrosis. No fungal structures were visualized and the new cultures were negative. After nine months, treatment was discontinued and the patient now has a 1-year clinical follow-up, sustaining complete remission of lesions (Figures 5-6).

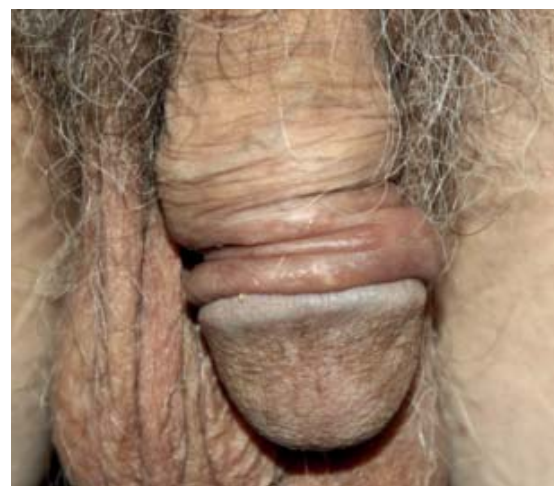

Figure 5:

Complete remission after treatment with oral itraconazole

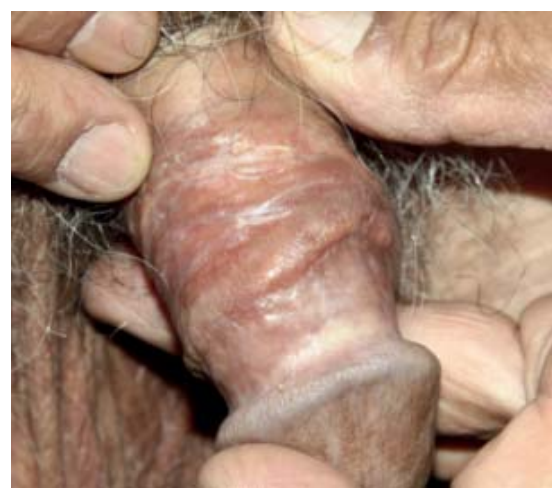

FIGURE 6:

Complete remission after treatment with oral itraconazole

\section{DISCUSSION}

Systemic mycoses with cutaneous involvement such as histoplasmosis, coccidioidomycosis and paracoccidioidomycosis are usually acquired by pulmonary inoculation through inhalation, and later disseminated to other organs such as the skin. The emergence of isolated skin lesions leads to the possibility of primary cutaneous inoculation, which is difficult to prove. ${ }^{4}$ Reports of genital ulcers caused by Histoplasma capsulatum are rare and most reports of cutaneous histoplasmosis occurred in immunosuppressed patients. ${ }^{5}$ The inoculation process in this report is not clear, since the patient doesn't refer previous genital trauma. Dust rich in spores that might be held in clothes after cleaning contaminated sites is a hypothe- 
sis that should be explored.

Despite the absence of immunosuppression signs, the elderly are associated with lower function of cellular immune response as well as worse vigilance against cancer and infectious diseases. ${ }^{6}$

Genital dermatoses comprise several diseases whose clinical manifestations can differ from their elsewhere presentations. Sexually transmitted diseases such as syphilis, chancroid and genital herpes may present as ulcerous lesions with adenopathy; neoplasms, inflammatory such as Behçet disease, traumatic and infectious diseases can have genital manifestations which dermatologists must be aware of.

We report the present case due to its very rare presentation of primary cutaneous histoplasmosis affecting a patient without evidence of immunosuppression or trauma.

\title{
REFERENCES
}

1. Ferreira MS, Borges AS. Histoplasmosis. Rev Soc Bras Med Trop. 2009;42:192-8.

2. Krunic AL, Carag H, Medenica MM, Lorincz AL. A Case of Cutaneous Histoplasmosis in a Patient with Diabetes and Multi-Infarct Dementia. J Dermatol. 2002:29:797-802.

3. Saheki MN, Schubach A0, Salgueiro MM, Conceição-Silva F, Wanke B, Lazera M. Primary cutaneous histoplasmosis: case report on an immunocompetent patient and review of the literature. Rev Soc Bras Med Trop. 2008;41:680-2.

4. Tesh RB, Schneidau Jr JD. Primary cutaneous histoplasmosis. N Engl J Med. 1966 Sep 15;275:597-9.

5. de Oliveira EV, Miduati FB, Antonio JR, de Negreiros Moraes R, de Almeida MT, D'Avilla SC, et al. Histoplasmosis as cause of penile ulcer in acquired immune deficiency syndrome (AIDS): three case reports. Mycopathologia. 2007;164:295-9.

6. Butcher SK, Killampalli V, Chahal H, Kaya Alpar E, Lord JM. Effect of age on susceptibility to post-traumatic infection in the elderly. Biochem Soc Trans 2003;31:449-51.

\author{
MAILING ADDRESS: \\ João de Magalhães Avancini Ferreira Alves \\ Av. Dr. Enéas de Carvalho Aguiar, 255, sala 3068 \\ 05403-900 - Sao Paulo - SP \\ Brazil \\ E-mail: joao.avancini@hc.fm.usp.br
}

How to cite this article: Paixão M, Miot HA, Avancini J, Belda Junior W. Primary cutaneous histoplasmosis developed in the penis of an immunocompetent patient. An Bras Dermatol. 2015;90(2):255-7. 